\title{
Multi-scale Analysis of SPDEs with Degenerate Additive Noise
}

\author{
Wael W. Mohammed ${ }^{1}$, Dirk Blömker ${ }^{2}$ and Konrad Klepel ${ }^{2}$ \\ ${ }^{1}$ Department of Mathematics, \\ Faculty of Science, \\ Mansoura University, Egypt \\ E-mail: wael.mohammed@mans.edu.eg \\ ${ }^{2}$ Institut für Mathematik \\ Universität Augsburg, Germany \\ E-mail: dirk.bloemker@math.uni-augsburg.de and \\ konrad.klepel@math.uni-augsburg.de
}

November 5, 2013

\begin{abstract}
We consider a quite general class of SPDEs with quadratic and cubic nonlinearities and derive rigorously amplitude equations, using the natural separation of time-scales near a change of stability. We show that degenerate additive noise has the potential to stabilize or destabilize the dynamics of the dominant modes, due to additional deterministic terms arising in averaging.

We focus on equations with quadratic and cubic nonlinearities and give applications to the Burgers' equation, the Ginzburg-Landau equation and generalized Swift-Hohenberg equation.
\end{abstract}

Keywords: Amplitude equation, multi-scale analysis, stabilization, stochastic partial differential equations, degenerate noise

MSC2010: 60H15, 60H10

\section{Introduction}

Stochastic partial differential equations (SPDEs) appear in several applications, for instance the stochastic Swift-Hohenberg equation, which was first used as a toy model for the convective instability in Rayleigh-Bénard problem (see [7] or [16]), and stochastic Burgers' equation, which use in the study of closure models for hydrodynamic turbulence [8].

Here we consider parabolic nonlinear SPDEs with additive forcing close to a change of stability, where the order of the noise strength is comparable 
to the order of the distance from the change of stability. Under appropriate scaling close to bifurcation one can reduce the essential dynamics to a simpler model for the amplitudes of the dominant bifurcating modes.

The general prototype of equations under consideration is an equation of the type

$$
d u(t)=\left[\mathcal{A} u(t)+\varepsilon^{2} \mathcal{L} u(t)+B(u(t))+\mathcal{F}(u(t))\right] d t+\sigma_{\varepsilon} d W(t),
$$

where $\mathcal{A}$ is non-positive operator with finite dimensional kernel, $\varepsilon^{2} \mathcal{L} u$ is a small deterministic perturbation, $B(u)$ is a quadratic nonlinearity, $\mathcal{F}$ is a cubic nonlinearity, and $W$ is finite dimensional noise for simplicity.

Near a change of stability, we can rely on the natural separation of timescales, in order to derive simpler equations for the evolution of the dominant modes or pattern that change stability. As these equations describe the amplitude of these pattern, they are referred to as amplitude equations. As we are forcing only non-dominant modes, if the noise is too small there is no direct impact on the amplitude equation. But as soon as the distance to the change of stability is comparable to the order of the squared noise strength, we will see additional terms in the amplitude equation induced by the noise. This is due to the fact that the degenerate additive noise is transported via nonlinear interaction to the dominant pattern. Other examples of this effect are $[2,4,13,14,15,21,22]$. Related works in this direction are [19, 23].

The aim of this paper is to derive rigorously the amplitude equation for a quite general class of SPDEs (cf. (1)) and investigate whether additive degenerate noise (i.e. noise that does not act directly on the dominant mode) can lead to stabilization of the solution of the SPDE (1). We could easily treat even higher order terms like, for instance, quartic or quintic, but they do not have any impact on the final result. Thus we can think of $B$ and $\mathcal{F}$ as the lowest order terms in a Taylor expansion of a more general nonlinearity.

Interesting effects appear due the appearance of additional deterministic terms in the amplitude equation due to stochastic averaging. These terms can change the nature of stability of the dominant modes. Here we present two cases. In the first one due to noise and nonlinear interaction deterministic linear terms appear in the amplitude equation. In the second one a deterministic constant forcing term appears, and stabilizes the dominant pattern, as the dominant mode is driven away from zero. To our knowledge, this was not observed before.

In all our examples the noise strength $\sigma_{\varepsilon}$ scales with the distance from bifurcation. In experiments $\sigma_{\varepsilon}$ is usually fixed, while one is free to vary the distance from bifurcation given by $\varepsilon^{2}$. Nevertheless, here we take the equivalent viewpoint with $\varepsilon^{2}$ small but fixed, and consider different scalings of the small noise strength $\sigma_{\varepsilon}$.

The case of $\sigma_{\varepsilon}=\varepsilon^{2}$ was treated in [1] for cubic nonlinearities like SwiftHohenberg and in [3] for quadratic nonlinearities like Burgers equation. Here 
the dominant behavior is given by an SDE, called the amplitude equation, where the noise only acts directly on the dominant modes.

The case $\sigma_{\varepsilon}=\varepsilon$ is treated in [4] for cubic nonlinearities and in [2] for quadratic nonlinearities like Burgers equation, where only noise not acting directly on the dominant modes influences the final result. Due to averaging additional linear deterministic terms appear that have the potential to stabilize or destabilize the dominant behavior. In [17] a generalized SwiftHohenberg equation was studied with polynomial nonlinearity containing cubic and quadratic terms.

Here we revisit the case $\sigma_{\varepsilon}=\varepsilon$ and generalize the previously obtained results in $[2,4,17]$ in a unified framework. The new interesting case of noise strength $\sigma_{\varepsilon}=\varepsilon^{3 / 2}$ with noise not acting directly on the dominant modes leads to deterministic constant forcing in the amplitude equations. This was to our knowledge not studied before.

The rest of this paper is organized as follows. In Section 2 we state the precise setting for equation (1) and the assumptions that we need. In Section 3 we discuss the first reduction steps and state the main theorem. Section 4 gives bounds for high non-dominant modes. In Section 5 we give the proof of the approximation Theorem I and some applications like the Burgers' equation (treated also in [2]) the Ginzburg-Landau Equation (treated also in [4]) and the generalized Swift-Hohenberg equation (also treated in [17]). Finally, we prove the the approximation Theorem II and apply this result to the generalized Swift-Hohenberg equation.

\section{Setting \& Assumptions}

This section states the precise setting for (1) and summarizes all assumptions that are necessary for our results. For the analysis we will work in some separable Hilbert space $\mathcal{H}$ equipped with scalar product $\langle\cdot, \cdot\rangle$ and norm $\|\cdot\|$. For the linear operator $\mathcal{A}$ we assume the following:

Assumption 1 (Linear operator $\mathcal{A}$ ) Suppose $\mathcal{A}$ is a non-positive selfadjoint operator on $\mathcal{H}$ with real eigenvalues $-\lambda_{k}$ such that

$$
0=\lambda_{1}=\ldots=\lambda_{n}<\lambda_{n+1} \leq \ldots \leq \lambda_{k} \leq \ldots \quad \text { and } \quad \lambda_{k} \geq C k^{m}
$$

for all sufficiently large $k$ and one $m>0$. The corresponding eigenfunctions $\left\{e_{k}\right\}_{k=1}^{\infty}$ form a complete orthonormal system in $\mathcal{H}$ such that $\mathcal{A} e_{k}=-\lambda_{k} e_{k}$.

From the assumption, we know that $\mathcal{N}:=\operatorname{ker} \mathcal{A}$ has finite dimension $n$ with basis $\left(e_{1}, \ldots, e_{n}\right)$. Note that the growth rate of the eigenvalues $\lambda_{k}$ depends on the dimension. If $\mathcal{A}$ is a differential operator on functions on a bounded interval, as in our examples, then $m$ corresponds with the order of the operator. 
Define by $S=\mathcal{N}^{\perp}$ the orthogonal complement of $\mathcal{N}$ in $\mathcal{H}$, and by $P_{c}$ the orthogonal projection onto $\mathcal{N}$. Define the projection onto the orthogonal complement by $P_{s}:=\mathcal{I}-P_{c}$, where $\mathcal{I}$ is the identity operator on $\mathcal{H}$.

For $\alpha \in \mathbb{R}$, we define the space $\mathcal{H}^{\alpha}$ by Fourier series:

$\mathcal{H}^{\alpha}=\left\{\sum_{k=0}^{\infty} \gamma_{k} e_{k}: \sum_{k=1}^{\infty} k^{2 \alpha} \gamma_{k}^{2}<\infty\right\} \quad$ with norm $\quad\left\|\sum_{k=1}^{\infty} \gamma_{k} e_{k}\right\|_{\alpha}^{2}=\sum_{k=0}^{\infty} k^{2 \alpha} \gamma_{k}^{2}$.

Note that in our examples, with $\mathcal{A}$ being a polynomial of the Laplacian on an interval, the space $\mathcal{H}^{\alpha}$ coincides with the usual Sobolev space $H^{\alpha}$. If we also have an upper bound on the eigenvalues (i.e., $\lambda_{k} \sim k^{m}$ ), then $\mathcal{H}^{\alpha}$ can be seen as a fractional interpolation space.

The operator $\mathcal{A}$ given by Assumption 1 generates an analytic semigroup $\left\{e^{t \mathcal{A}}\right\}_{t \geq 0}$, (cf. Dan Henry [12] or Pazy [18]), on any space $\mathcal{H}^{\alpha}$ defined by

$$
e^{\mathcal{A} t}\left(\sum_{k=1}^{\infty} \gamma_{k} e_{k}\right)=\sum_{k=1}^{\infty} e^{-\lambda_{k} t} \gamma_{k} e_{k} \quad \forall t \geq 0
$$

and has the following property for all $t>0, \beta \geq \alpha, \lambda_{n}<\omega \leq \lambda_{n+1}$ and all $u \in \mathcal{H}^{\beta}$

$$
\left\|e^{t \mathcal{A}} P_{s} u\right\|_{\alpha} \leq M t^{-\frac{\alpha-\beta}{m}} e^{-\omega t}\left\|P_{s} u\right\|_{\beta},
$$

where $M$ depends only on the constants $\alpha, m, \beta$, and $\omega$.

Assumption 2 (Operator $\mathcal{L}$ ) Let $\mathcal{L}: \mathcal{H}^{\alpha} \rightarrow \mathcal{H}^{\alpha-\beta}$ for some $\alpha \in \mathbb{R}$ and some $\beta \in[0, m)$ be a linear continuous mapping that commutes with $P_{c}$ and $P_{s}$.

The Assumption on $\mathcal{L}$ basically states that the operator is a compact perturbation of the operator $\mathcal{A}: \mathcal{H}^{\alpha} \rightarrow \mathcal{H}^{\alpha}$. For details see Pazy [18] or Henry [12]. In the example of differential operators one can think of operators of lower order.

For the quadratic nonlinearity $B$ we make two assumptions. The first one was crucial in [2] and is satisfied for equations like the Burgers equation. It basically guarantees that a single mode cannot map back via the quadratic nonlinearity to the dominant mode.

Assumption 3 (Bilinear Operator $B$ - I) With $\alpha$ and $\beta$ from Assumption 2 let $B$ be a bounded symmetric bilinear mapping from $\mathcal{H}^{\alpha} \times \mathcal{H}^{\alpha}$ to $\mathcal{H}^{\alpha-\beta}$. Suppose that $P_{c} B\left(e_{k}, e_{k}\right)=0$ for all $k \in \mathbb{N}$.

The second assumption relaxes the first one, as it allows single nondominant modes to map back to the dominant ones. But dominant modes still cannot map back to the dominant mode. This is crucial, in order to study both cubic and quadratic nonlinearities with only cubic nonlinearities appearing in the Amplitude equation. 
Assumption 4 (Bilinear Operator B - II) With $\alpha$ and $\beta$ from Assumption 2 let $B$ be a bounded symmetric bilinear mapping from $\mathcal{H}^{\alpha} \times \mathcal{H}^{\alpha}$ to $\mathcal{H}^{\alpha-\beta}$. Suppose that $P_{c} B\left(e_{k}, e_{k}\right)=0$ for $k \in\{1,2, \ldots, n\}$, where $n$ is the dimension of the null-space of $\mathcal{A}$.

For the cubic nonlinearity $\mathcal{F}$ we assume that:

Assumption 5 Assume that $\mathcal{F}:\left(\mathcal{H}^{\alpha}\right)^{3} \rightarrow \mathcal{H}^{\alpha-\beta}$ with $\beta$ as in Assumption 2 is trilinear, symmetric and bounded. Thus for some $C>0$

$$
\|\mathcal{F}(u, v, \omega)\|_{\alpha-\beta} \leq C\|u\|_{\alpha}\|v\|_{\alpha}\|\omega\|_{\alpha} \quad \forall u, v, \omega \in \mathcal{H}^{\alpha},
$$

The assumption that $B$ and $\mathcal{F}$ are symmetric is without loss of generality. As they are quadratic and cubic we can always define them in a symmetric way.

Denote $B(u)=B(u, u)$ and $\mathcal{F}(u)=\mathcal{F}(u, u, u)$ for short. Moreover, we denote the projections by indices. This means $\mathcal{F}_{c}=P_{c} \mathcal{F}$ or $\mathcal{F}_{s}=P_{s} \mathcal{F}$. We define $B_{s}, B_{c}$, and $\mathcal{L}_{c}$ in a similar way.

For the noise we suppose:

Assumption 6 Let $W$ be a finite dimensional Wiener process on $\mathcal{H}$, such that for $t \geq 0$,

$$
W(t)=\sum_{k} \alpha_{k} \beta_{k}(t) e_{k} \quad \text { for finitely many } k \geq n+1,
$$

where $\left(\beta_{k}\right)_{k}$ are independent, standard Brownian motions in $\mathbb{R}$ and $\left(\alpha_{k}\right)_{k}$ are real numbers.

The assumption of finiteness on the noise is mainly for simplicity of presentation. We could handle infinite sums, but would need in this case several conditions for series to converge. Moreover, the fact that the noise is given as a Fourier series with respect to the $e_{k}$ is not important. But it is a key assumption, that the noise is degenerate, i.e., that $P_{c} W=0$.

The following assumption is crucial in order to obtain long-time results and global existence for the amplitude equation.

Assumption 7 Define the cubic nonlinearity $\tilde{F}: \mathcal{N} \rightarrow \mathcal{N}$ via

$$
\tilde{F}(a)=-2 B_{c}\left(a, \mathcal{A}_{s}^{-1} B_{s}(a, a)\right)+\mathcal{F}_{c}(a) .
$$

Assume there is a constant $c_{0} \geq 0$ and a constant $C \geq 0$ such that for all $a, b, R \in \mathcal{N}$

$$
\langle\tilde{F}(a+b+R)-\tilde{F}(b), a\rangle \leq-c_{0}|a|^{4}+C|R|^{4}+C|R|^{2}|b|^{2} .
$$

Setting $b=0=R$ we immediately obtain: 
Corollary 8 Under Assumption 7 for all $a \in \mathcal{N}$

$$
\langle\tilde{F}(a), a\rangle \leq-c_{0}|a|^{4} .
$$

Example 9 For the standard cubic nonlinearity $\tilde{F}(a)=-|a|^{2} a$ Assumption 7 is true with positive $c_{0}>0$. Note that here $3 \tilde{F}(a, b, d)=-(a \cdot b) d-(a$. $d) b-(d \cdot b) a$.

To give a meaning to (1) we always consider mild solutions.

Definition 10 (Local mild solution) An $\mathcal{H}^{\alpha}$-valued process $\{u(t)\}_{t \in[0, T]}$ is a local mild solution of (1) if for some stopping time $\tau_{0}$ we have on a set of probability 1 that $\tau_{0}>0$ and $u \in C^{0}\left(\left[0, \tau_{0}\right], \mathcal{H}^{\alpha}\right)$ such that

$u(t)=e^{t \mathcal{A}} u_{0}+\int_{0}^{t} e^{(t-s) \mathcal{A}}\left[\varepsilon^{2} \mathcal{L} u+B(u)+\mathcal{F}(u)\right] d s+\sigma_{\varepsilon} W_{\mathcal{A}}(t), \quad \forall t \in\left(0, \tau_{0}\right], \quad \mathbb{P}-a . s$,

where $W_{\mathcal{A}}$ is the stochastic convolution defined as

$$
W_{\mathcal{A}}(t)=\int_{0}^{t} e^{(t-s) \mathcal{A}} d W(s) .
$$

The existence and uniqueness of local solutions in the sense of the previous definition is standard, as we consider locally Lipschitz-continuous nonlinearities. See, e.g., [9] or [20] for many examples. Moreover, path-wise for each realization of randomness, the deterministic theory based on Banach's fixed point theorem can be applied (see [12], for example). It it is a well-known result, both in the deterministic and completely analogous in the stochastic case, that the solution $u$ and the stopping time $\tau_{0}$ can be extended such that $u \in C^{0}\left(\left[0, \tau_{0}\right), \mathcal{H}^{\alpha}\right)$ and either $\tau_{0}=\infty$ or $\|u(t)\|_{\alpha} \rightarrow \infty$ for $t \rightarrow \tau_{0}$. See for example Theorem 2.2 of [5].

Let us also remark that in the definition $\tau_{0}$ could only be a random time, but here we focus on stopping times with respect to the canonical filtration induced by $W$.

For our result we rely on a cut off argument. First we consider only solutions $u$ that are not too large, as given by the stopping time below. Thus we can always control the Lipschitz-constants of the nonlinear terms and higher moments of the nonlinearity. Later, we use the amplitude equations, in order to show that the stopping time is actually large, at least with high probability.

Definition 11 For a mild solution $u$ of (1) we define, for some fixed $T_{0}>0$ and small $\kappa \in\left(0, \frac{1}{18}\right)$, the stopping time $\tau^{*}$, with respect to the canonical filtration induced by $W$, by

$$
\tau^{*}:=T_{0} \wedge \inf \left\{T>0:\left\|u\left(T \varepsilon^{-2}\right)\right\|_{\alpha}>\varepsilon^{1-\kappa}\right\} .
$$


Definition 12 For a real-valued family of processes $\left\{X_{\varepsilon}(t)\right\}_{t \geq 0}$ we say $X_{\varepsilon}=\mathcal{O}\left(f_{\varepsilon}\right)$, if for every $p \geq 1$ there exists a constant $C_{p}$ such that

$$
\mathbb{E} \sup _{t \in\left[0, \tau^{*}\right]}\left|X_{\varepsilon}(t)\right|^{p} \leq C_{p} f_{\varepsilon}^{p} .
$$

We use also the analogous notation for time-independent random variables.

\section{Derivation and Main Results}

In this section we present the first reduction step and state the main results. We are interested here in studying behavior of solution to (1) on the long time-scale of order $\varepsilon^{-2}$ induced by the distance from bifurcation. Furthermore, due to the noise-strength and the distance from bifurcation, we cannot expect solutions to be too large. Finally, our aim is to obtain a reduced equation for the evolution of the dominant modes.

Thus we rescale and split the solution $u$ into

$$
u(t)=\varepsilon a\left(\varepsilon^{2} t\right)+\varepsilon \psi\left(\varepsilon^{2} t\right),
$$

where $a$ is an $\mathcal{N}$-valued and $\psi$ an $S$-valued process. By the definition (6) of $\tau^{*}$ we have that

$$
\|a(T)\|_{\alpha} \leq \varepsilon^{-\kappa} \quad \text { and } \quad\|\psi(T)\|_{\alpha} \leq \varepsilon^{-\kappa} \quad \text { for all } T \leq \tau^{*} .
$$

After rescaling to the slow time-scale $T=\varepsilon^{2} t$, we obtain the following system of equations:

$$
d a=\left[\mathcal{L}_{c} a+2 \varepsilon^{-1} B_{c}(a, \psi)+\varepsilon^{-1} B_{c}(\psi)+\mathcal{F}_{c}(a+\psi)\right] d T,
$$

and

$$
d \psi=\left[\varepsilon^{-2} \mathcal{A}_{s} \psi+\mathcal{L}_{s} \psi+\varepsilon^{-1} B_{s}(a+\psi)+\mathcal{F}_{s}(a+\psi)\right] d T+\sigma_{\varepsilon} \varepsilon^{-2} d \tilde{W},
$$

where $\tilde{W}(T):=\varepsilon W\left(\varepsilon^{-2} T\right)$ is a rescaled version of the Wiener process.

Equation (9) reads in the integrated form

$$
a(T)=a(0)+\int_{0}^{T} \mathcal{L}_{c} a d \tau+\varepsilon^{-1} \int_{0}^{T} B_{c}(2 a+\psi, \psi) d \tau+\int_{0}^{T} \mathcal{F}_{c}(a+\psi) d \tau .
$$

In order to obtain the amplitude equation in $a$ only, we need two steps. As a first step, we have to remove all terms explicitly depending on $\varepsilon^{-1}$, as these might not be small. In a second step, we use stochastic averaging in order to get rid of all the $\psi$ from Equation (9).

As the first step, we start with removing the $\varepsilon^{-1}$. This can be achieved by Itô's formula applied to $B_{c}\left(a, \mathcal{A}_{s}^{-1} \psi\right)$ and to $B_{c}\left(e_{k}, e_{\ell}\right) \psi_{k} \psi_{\ell}$. Recall that $\mathcal{A}_{s}^{-1}$ is well defined on $P_{s} \mathcal{H}$. We obtain for the first term 


$$
\begin{aligned}
\varepsilon^{-1} & \int_{0}^{T} B_{c}(a, \psi) d \tau=\varepsilon B_{c}\left(a(T), \mathcal{A}_{s}^{-1} \psi(T)\right)-\varepsilon B_{c}\left(a(0), \mathcal{A}_{s}^{-1} \psi(0)\right) \\
& -\varepsilon \int_{0}^{T} B_{c}\left(\mathcal{L}_{c} a, \mathcal{A}_{s}^{-1} \psi\right) d \tau-\int_{0}^{T} B_{c}\left(B_{c}(2 a+\psi, \psi), \mathcal{A}_{s}^{-1} \psi\right) d \tau \\
& -\int_{0}^{T} B_{c}\left(a, \mathcal{A}_{s}^{-1} B_{s}(a, a+2 \psi)\right) d \tau-\varepsilon \int_{0}^{T} B_{c}\left(\mathcal{F}_{c}(a+\psi), \mathcal{A}_{s}^{-1} \psi\right) d \tau \\
& -\int_{0}^{T} B_{c}\left(a, \mathcal{A}_{s}^{-1} B_{s}(\psi, \psi)\right) d \tau-\sigma_{\varepsilon} \varepsilon^{-1} \int_{0}^{T} B_{c}\left(a, \mathcal{A}_{s}^{-1} d \tilde{W}_{s}\right) \\
& -\varepsilon \int_{0}^{T} B_{c}\left(a, \mathcal{A}_{s}^{-1} \mathcal{L}_{s} \psi\right) d \tau-\varepsilon \int_{0}^{T} B_{c}\left(a, \mathcal{A}_{s}^{-1} \mathcal{F}_{s}(a+\psi)\right) d \tau,
\end{aligned}
$$

and for the second (assuming all sums run over the forced modes $k, \ell \geq n+1$ )

$$
\begin{aligned}
\varepsilon^{-1} \int_{0}^{T} B_{c}(\psi, \psi) d \tau=\varepsilon^{-1} \sum_{\ell, k} B_{c}\left(e_{k}, e_{\ell}\right) \int_{0}^{T} \psi_{k} \psi_{\ell} d \tau \\
=-\varepsilon \sum_{\ell, k} \frac{B_{c}\left(e_{k}, e_{\ell}\right)}{\left(\lambda_{k}+\lambda_{\ell}\right)}\left[\psi_{k}(T) \psi_{\ell}(T)-\psi_{k}(0) \psi_{\ell}(0)\right] \\
\quad+\varepsilon \sum_{\ell, k} \frac{2 B_{c}\left(e_{k}, e_{\ell}\right)}{\left(\lambda_{k}+\lambda_{\ell}\right)} \int_{0}^{T}\left[\left\langle\mathcal{L}_{s} \psi, e_{\ell}\right\rangle+\mathcal{F}_{\ell}(a+\psi)\right] \psi_{k} d \tau \\
\quad+\sum_{\ell, k} \frac{2 B_{c}\left(e_{k}, e_{\ell}\right)}{\left(\lambda_{k}+\lambda_{\ell}\right)}\left[\sigma_{\varepsilon} \varepsilon^{-1} \alpha_{k} \int_{0}^{T} \psi_{\ell} d \tilde{\beta}_{k}+\int_{0}^{T} B_{\ell}(a+\psi) \psi_{k} d \tau\right] \\
\quad+\frac{1}{4} \sigma_{\varepsilon}^{2} \varepsilon^{-3} \sum_{k} \frac{\alpha_{k}^{2}}{\lambda_{k}} B_{c}\left(e_{k}, e_{k}\right) T .
\end{aligned}
$$

Here $B_{\ell}(w)=\left\langle B(w), e_{\ell}\right\rangle$. Substituting from (12) and (13) into (11) we obtain

$$
\begin{aligned}
a(T)= & a(0)+\int_{0}^{T}\left[\mathcal{L}_{c} a+\tilde{F}(a)-2 B_{c}\left(B_{c}(\psi, \psi), \mathcal{A}_{s}^{-1} \psi\right)\right] d \tau \\
& -\int_{0}^{T}\left[4 B_{c}\left(B_{c}(a, \psi), \mathcal{A}_{s}^{-1} \psi\right)+4 B_{c}\left(a, \mathcal{A}_{s}^{-1} B_{s}(a, \psi)\right)\right] d \tau \\
& -\int_{0}^{T}\left[2 B_{c}\left(a, \mathcal{A}_{s}^{-1} B_{s}(\psi, \psi)\right)-3 \mathcal{F}_{c}(a, a, \psi)\right] d \tau \\
& +\int_{0}^{T}\left[3 \mathcal{F}_{c}(a, \psi, \psi)+\mathcal{F}_{c}(\psi)\right] d \tau-2 \sigma_{\varepsilon} \varepsilon^{-1} \int_{0}^{T} B_{c}\left(a, \mathcal{A}_{s}^{-1} d \tilde{W}\right) \\
& +\sum_{\ell, k} \frac{2 B_{c}\left(e_{k}, e_{\ell}\right)}{\left(\lambda_{k}+\lambda_{\ell}\right)}\left[\sigma_{\varepsilon} \varepsilon^{-1} \alpha_{k} \int_{0}^{T} \psi_{\ell} d \tilde{\beta}_{k}+\int_{0}^{T} B_{\ell}(a+\psi) \psi_{k} d \tau\right]
\end{aligned}
$$




$$
+\frac{1}{4} \sigma_{\varepsilon}^{2} \varepsilon^{-3} \sum_{k} \frac{\alpha_{k}^{2}}{\lambda_{k}} B_{c}\left(e_{k}, e_{k}\right) T+\mathcal{O}\left(\varepsilon^{1-4 \kappa}\right)
$$

where the cubic term $\tilde{F}(a)$ is given by (4).

\subsection{The scaling $\sigma_{\varepsilon}=\varepsilon$.}

The first main result of this paper is the rigorous derivation of an amplitude equation in the case of $\sigma_{\varepsilon}=\varepsilon$. For simplicity we assume that the noise is forcing only one mode called $k$. If not, we get all terms we have with sums and several additional terms (see Remark 14 below). In the single mode case the amplitude equation reads

$$
\partial_{T} b=\mathcal{L}_{c} b+\tilde{F}(b)+\tilde{G} b+\frac{2 \alpha_{k}}{\lambda_{k}} B_{c}\left(b, e_{k}\right) \partial_{T} \tilde{\beta}_{k},
$$

with a linear map $\tilde{G}: \mathcal{N} \rightarrow \mathcal{N}$ given by

$$
\begin{aligned}
\tilde{G} b= & \frac{3 \alpha_{k}^{2}}{2 \lambda_{k}} \mathcal{F}_{c}\left(b, e_{k}, e_{k}\right)-\frac{\alpha_{k}^{2}}{\lambda_{k}} B_{c}\left(b, \mathcal{A}_{s}^{-1} B_{s}\left(e_{k}, e_{k}\right)\right) \\
& +\frac{2 \alpha_{k}^{2}}{\lambda_{k}^{2}} B_{c}\left(B_{c}\left(b, e_{k}\right), e_{k}\right)+\sum_{\ell \neq k} \frac{2 \alpha_{k}^{2} B_{\ell}\left(b, e_{k}\right)}{\lambda_{k}\left(\lambda_{k}+\lambda_{\ell}\right)} B_{c}\left(e_{\ell}, e_{k}\right),
\end{aligned}
$$

where $B_{\ell}=P_{\ell} B$ with projection $P_{\ell}: \mathcal{H}^{\alpha} \rightarrow \mathbb{R}$ defined by $P_{\ell}\left(\sum_{k} \alpha_{k} e_{k}\right)=\alpha_{\ell}$.

We show in our main Theorem I that near a change of stability on a time-scale of order $\varepsilon^{-2}$ the solution of (1) is well estimated by

$$
u(t) \simeq \varepsilon b\left(\varepsilon^{2} t\right)+\varepsilon \mathcal{Z}_{k}\left(\varepsilon^{2} t\right) e_{k}+\mathcal{O}\left(\varepsilon^{2-}\right),
$$

where $b$ is the solution of the amplitude equation (17) and the fast realvalued $\mathrm{OU}$ process $\mathcal{Z}_{k}(T)$ is defined by

$$
\mathcal{Z}_{k}(T):=\alpha_{k} \varepsilon^{-1} \int_{0}^{T} e^{-\varepsilon^{-2} \lambda_{k}(T-\tau)} d \tilde{\beta}_{k}(\tau),
$$

with $\tilde{\beta}_{k}(T):=\varepsilon \beta_{k}\left(\varepsilon^{-2} T\right)$ being a rescaled version of the Brownian motion.

The main result in this case is:

Theorem 13 (Approximation I) Let Assumptions 1, 2, 3, 5, 6, and 7 be true, where only one mode $k$ is forced. Let $u$ be a solution of (1) defined in (8) with the initial condition $u(0)$ of order $\varepsilon$ and split $u(0)=\varepsilon a(0)+\varepsilon \psi(0)$ with $a(0) \in \mathcal{N}$ and $\psi(0) \in S$ where $a(0)$ and $\psi(0)$ are of order one. Suppose $b$ is a solution of (15) with $b(0)=a(0)$. Then for all $p>1$ and $T_{0}>0$ and all $\kappa \in\left(0, \frac{1}{12}\right)$, there exists $C>0$ such that

$$
\mathbb{P}\left(\sup _{t \in\left[0, \varepsilon^{-2} T_{0}\right]}\left\|u(t)-\varepsilon b\left(\varepsilon^{2} t\right)-\varepsilon e^{t \mathcal{A}_{s}} \psi(0)-\varepsilon \mathcal{Z}_{k}\left(\varepsilon^{2} t\right) e_{k}\right\|_{\alpha}>\varepsilon^{2-13 \kappa}\right) \leq C \varepsilon^{p},
$$

where $\mathcal{Z}_{k}$ defined in (18). 
Remark 14 For simplicity we forced the noise in the above theorem in one mode only. If we force the noise in many modes we will have nonlinear interactions leading to a martingale term. One example are integrals of the type $\int_{0}^{T} Z_{\ell} d \beta_{k}$. In order to approximate this martingale term, we need to use Lemma 6.1 from [2], which is strictly only for one-dimensional $\mathcal{N}$, as it is based on Levy representation and the martingale representation theorem. For more related results in this direction, see for instance [4].

Remark 15 Let us comment on the case, when Assumption 7 fails to be true. In that case we do not obtain control on the stopping time $\tau^{*}$. Especially, $\tau^{*}=T_{0}$ might have very small probability due to blow up in finite time of the amplitude equation. Nevertheless in the proof we still establish a bound like:

$$
\mathbb{E} \sup _{t \in\left[0, \varepsilon^{-2} \tau^{*}\right]}\left\|u(t)-\varepsilon b\left(\varepsilon^{2} t\right)-\varepsilon e^{t \mathcal{A}_{s}} \psi(0)-\varepsilon \mathcal{Z}_{k}\left(\varepsilon^{2} t\right) e_{k}\right\|_{\alpha}^{p} \leq C \varepsilon^{p(1-12 \kappa)} .
$$

To illustrate our approximation result of Theorem 13 we consider here the setting of [17], which is a stochastic Swift-Hohenberg equation with respect to periodic boundary conditions on $[0,2 \pi]$ and forced by spatially constant noise:

$$
\partial_{t} u=-\left(1+\partial_{x}^{2}\right)^{2} u+\nu \varepsilon^{2} u+\gamma u^{2}-u^{3}+\frac{1}{\sqrt{2 \pi}} \varepsilon \sigma \partial_{t} \beta .
$$

The approximation theorem in this case states that the solution of (21) is of the type

$$
u(t)=\varepsilon v\left(\varepsilon^{2} t\right)
$$

with

$$
v(T)=b_{1}(T) \sin +b_{-1}(T) \cos +\sigma Z_{0}+\mathcal{O}\left(\varepsilon^{1-}\right),
$$

where the fast OU-process $Z_{0} \simeq \partial_{T} \tilde{\beta}(T)$ is well approximated by noise, and $b_{1}$ and $b_{-1}$ are the solutions of the amplitude equation

$d b_{i}=\left[\left(\nu-\frac{3}{2} \sigma^{2}+3 \sigma^{2} \gamma^{2}\right) b_{i}+\frac{3}{4}\left(\frac{38}{27} \gamma^{2}-1\right) b_{i}\left(b_{1}^{2}+b_{-1}^{2}\right)\right] d T+2 \gamma \sigma b_{i} d \tilde{\beta}_{0}$ for $i= \pm 1$.

Here we have to assume that $\frac{27}{38}>\gamma^{2}$. Otherwise the nonlinearity is not stable, and Assumption 7 fails to be true. In that case we cannot apply our theorems directly. In case $\frac{27}{38}<\gamma^{2}$ the result would only hold up to a possible blow-up time of the amplitude equation. An interesting scaling is the case $\frac{27}{38}=\gamma^{2}$. Here the amplitude equation is linear, and we could consider larger solutions, and still obtain a meaningful result. The amplitude equation in that case will have quintic nonlinearities. This case was studied by [6] for the deterministic equation.

If we choose $\gamma \leq \frac{1}{2}$ and $\sigma^{2} \geq \frac{4 \nu}{3}$, then the constant $\left(\nu-\frac{3}{2} \sigma^{2}+3 \sigma^{2} \gamma^{2}\right)$ in front of the linear term is negative. In this case we can say that degenerate additive noise has the potential to stabilize the dynamics of the dominant modes. 


\subsection{The scaling $\sigma_{\varepsilon}=\varepsilon^{3 / 2}$}

The second approximation result of this paper considers the case where $\sigma_{\varepsilon}=\varepsilon^{\frac{3}{2}}$. Here we allow for many modes being forced, and for nonlinear interaction of the noise terms. The first approximation result still holds, as the noise strength is an order $\varepsilon^{1 / 2}$ smaller than before. But we will loose all the impact of the noise in the amplitude equation. Thus we allow for a different assumption on the nonlinearity (i.e., $B_{c}\left(e_{k}, e_{k}\right) \neq 0$ for nondominant and forced modes $k$.

The general result is the same as in the previous scaling, but the amplitude now takes the form

$$
d b(T)=\left[\mathcal{L}_{c} b+\tilde{F}(b)+\frac{1}{4} \sum_{k} \frac{\alpha_{k}^{2}}{\lambda_{k}} B_{c}\left(e_{k}, e_{k}\right)\right] d T .
$$

It is an interesting feature, that despite of the presence of noise, the amplitude equation is deterministic. Nevertheless, induced by the noise there is an additional constant forcing term, that will always drive solutions away from 0 . Thus already a little bit of noise will stabilize the dominant pattern, and prevents it from disappearing.

We prove the following theorem:

Theorem 16 (Approximation II) Under Assumptions 1, 2, 4, 5, 6, and 7 , let $u$ be a solution of (1) defined in (8) with the initial condition $u(0)=$ $\varepsilon a(0)+\varepsilon \psi(0)$ with $a(0) \in \mathcal{N}$ and $\psi(0) \in S$ where $a(0)$ and $\psi(0)$ are of order one, and $b$ is a solution of (22) with $b(0)=a(0)$. Then for all $p>1$ and $T_{0}>0$ and all $\kappa \in\left(0, \frac{1}{18}\right)$, there exists $C>0$ such that

$$
\mathbb{P}\left(\sup _{t \in\left[0, \varepsilon^{-2} T_{0}\right]}\left\|u(t)-\varepsilon b\left(\varepsilon^{2} t\right)-\varepsilon e^{t \mathcal{A}_{s}} \psi(0)\right\|_{\alpha}>\varepsilon^{3 / 2-10 \kappa}\right) \leq C \varepsilon^{p} .
$$

We see that the part depending on $\psi(0)$ decays exponentially fast on time-scales of order $\mathcal{O}\left(\varepsilon^{2}\right)$. Theorem 16 states that the solution of Equation (1) is well approximated by

$$
u(t) \simeq \varepsilon b\left(\varepsilon^{2} t\right)+\mathcal{O}\left(\varepsilon^{3 / 2-}\right),
$$

where $b$ is the solution of the amplitude equation in (22).

To illustrate our result, let us give an example of Theorem 16. Consider again the stochastic generalized Swift-Hohenberg equation with respect to periodic boundary conditions on $[0,2 \pi]$

$$
\partial_{t} u=-\left(4+\partial_{x}^{2}\right)^{2} u+\nu \varepsilon^{2} u+\gamma u^{2}-u^{3}+\sigma \varepsilon^{3 / 2} \partial_{t} \beta \cos (x) .
$$

Thus the dominant modes are $\mathcal{N}=\operatorname{span}(\sin (2 x), \cos (2 x))$. Obviously, the forced mode $\cos (x)$ is mapped via $u^{2}$ into $\mathcal{N}$. 
Our main theorem in this case states that the solution

$$
u(t)=\varepsilon v\left(\varepsilon^{2} t\right)
$$

of (25) is given by

$$
v(T, x) \simeq b_{2}(T) \sin (2 x)+b_{-2}(T) \cos (2 x)+\mathcal{O}\left(\varepsilon^{1 / 2-}\right),
$$

where $b_{2}$ and $b_{-2}$ are the solution of the amplitude equation

$$
\begin{aligned}
b_{2}^{\prime} & =\nu b_{2}-\left(\frac{3}{4}-\frac{5 \gamma^{2}}{72}\right) b_{2}\left(b_{2}^{2}+b_{-2}^{2}\right), \\
b_{-2}^{\prime} & =\nu b_{-2}-\left(\frac{3}{4}-\frac{5 \gamma^{2}}{72}\right) b_{-2}\left(b_{2}^{2}+b_{-2}^{2}\right)+\frac{\gamma \sigma^{2}}{72} .
\end{aligned}
$$

In this case it is essential that we choose $\gamma^{2}<\frac{54}{5}$, in order to have the coefficient in front of the cubic term negative. Otherwise our stability condition on $\tilde{F}$ would not be satisfied, and Assumption 7 fails to be true. See also the previous section for a similar discussion.

\section{Bounds for the high modes}

In this section we show that the non-dominant modes are already given by an OU-process and a contribution from the initial condition that dies out very fast.

Lemma 17 Under Assumptions 1, 2, 3 and 5, there is a constant $C>0$ such that, for $\kappa>0$ from the definition of $\tau^{*}$ and $p \geq 1$,

$$
\mathbb{E} \sup _{T \in\left[0, \tau^{*}\right]}\|\psi(T)-\mathcal{Q}(T)\|_{\alpha}^{p} \leq C \varepsilon^{p-3 p \kappa},
$$

where $\mathcal{Q}(T)$ is defined as

$$
\mathcal{Q}(T)=e^{\varepsilon^{-2} T \mathcal{A}_{s}} \psi(0)+\sigma_{\varepsilon} \varepsilon^{-1} \mathcal{Z}(T)
$$

Proof. Define

$$
\mathcal{Z}(T):=\sum_{k} \mathcal{Z}_{k}(T) e_{k},
$$

where $\mathcal{Z}_{k}(T)$ is defined in (18), where the sum runs over all forced modes. The mild solution of (10) is

$$
\begin{aligned}
\psi(T)= & e^{\varepsilon^{-2} T \mathcal{A}_{s}} \psi(0)+\int_{0}^{T} e^{\varepsilon^{-2}(T-\tau) \mathcal{A}_{s}}\left[\mathcal{L}_{s} \psi+\varepsilon^{-1} B_{s}(a+\psi)\right] d \tau \\
& \left.+\int_{0}^{T} e^{\varepsilon^{-2}(T-\tau) \mathcal{A}_{s}} \mathcal{F}_{s}(a+\psi)\right] d \tau+\sigma_{\varepsilon} \varepsilon^{-1} \mathcal{Z}(T)
\end{aligned}
$$


Using triangle inequality

$$
\begin{aligned}
\|\psi(T)-\mathcal{Q}(T)\|_{\alpha} \leq & \left\|\int_{0}^{T} e^{\varepsilon^{-2} \mathcal{A}_{s}(T-\tau)} \mathcal{L}_{s} \psi(\tau) d \tau\right\|_{\alpha} \\
& +\varepsilon^{-1}\left\|\int_{0}^{T} e^{\varepsilon^{-2} \mathcal{A}_{s}(T-\tau)} B_{s}(a+\psi) d \tau\right\|_{\alpha} \\
\quad & \quad\left\|\int_{0}^{T} e^{\varepsilon^{-2} \mathcal{A}_{s}(T-\tau)} \mathcal{F}_{s}(a+\psi) d \tau\right\|_{\alpha} \\
:= & I_{1}+I_{2}+I_{3} .
\end{aligned}
$$

We now bound these three terms separately. For the first term, we obtain by using (2) and Assumption 2 for all $T \leq \tau^{*}$

$$
\begin{aligned}
I_{1} & \leq C \varepsilon^{\frac{2 \beta}{m}} \int_{0}^{T} e^{-\varepsilon^{-2} \omega(T-\tau)}(T-\tau)^{-\frac{\beta}{m}}\|\psi(\tau)\|_{\alpha} d \tau \\
& \leq C \varepsilon^{2} \sup _{\tau \in\left[0, \tau^{*}\right]}\|\psi(\tau)\|_{\alpha} \int_{0}^{\varepsilon^{-2} \omega T} e^{-\eta} \eta^{-\frac{\beta}{m}} d \eta \\
& \leq C \varepsilon^{2-\kappa}
\end{aligned}
$$

where we used the definition of $\tau^{*}$. For the second term, we obtain completely similar by using (2) and Assumption 3 for all $T \leq \tau^{*}$

$$
\begin{aligned}
I_{2} & \leq C \varepsilon^{\frac{2 \beta}{m}-1} \int_{0}^{T} e^{-\varepsilon^{-2} \omega(T-\tau)}(T-\tau)^{-\frac{\beta}{m}}\|a+\psi\|_{\alpha}^{2} d \tau \\
& \leq C \varepsilon \sup _{\tau \in\left[0, \tau^{*}\right]}\|a+\psi\|_{\alpha}^{2} \int_{0}^{\varepsilon^{-2} \omega T} e^{-\eta} \eta^{-\frac{\beta}{m}} d \eta \\
& \leq C \varepsilon^{1-2 \kappa} .
\end{aligned}
$$

Analogously, for the third term. We obtain by using (2) and Assumption 5 that for all $T \leq \tau^{*}$

$$
I_{3} \leq C \varepsilon^{2-3 \kappa}
$$

Combining all results, yields (26).

Lemma 18 Under Assumption 1 and 6 , for every $\kappa_{0}>0$ and $p \geq 1$, there is a constant $C$, depending on $p, \alpha_{k}, \lambda_{k}, \kappa_{0}$ and $T_{0}$, such that

$$
\mathbb{E} \sup _{T \in\left[0, T_{0}\right]}\left|\mathcal{Z}_{k}(T)\right|^{p} \leq C \varepsilon^{-\kappa_{0}},
$$

and

$$
\mathbb{E} \sup _{T \in\left[0, T_{0}\right]}\|\mathcal{Z}(T)\|_{\alpha}^{p} \leq C \varepsilon^{-\kappa_{0}},
$$

where $\mathcal{Z}_{k}$ is defined in (18) and the finite sum $\mathcal{Z}$ in (28). 
Proof. This is a straightforward bound on fast OU-processes. See for instance the proof of Lemma 14 in [4]. With some more effort the bound should be logarithmic in $\varepsilon$.

The following Corollary states that $\psi(T)$ is with high probability much smaller than $\varepsilon^{-\kappa}$ as asserted by the Definition 11 for $T \leq \tau^{*}$. We use this later in order to show that $\tau^{*} \geq T_{0}$ with high probability (cf. Proof of Theorem 13).

Corollary 19 Under the assumptions of Lemmas 17 and 18, if $\psi(0)=$ $\mathcal{O}(1)$, then for $p>0$ and for all $\kappa_{0}>0$ there exist a constant $C>0$ such that

$$
\mathbb{E} \sup _{T \in\left[0, \tau^{*}\right]}\|\psi(T)\|_{\alpha}^{p} \leq C \varepsilon^{-\kappa_{0}}
$$

Proof. We use

$$
\|\psi\|^{p} \leq C\|\psi-\mathcal{Q}\|^{p}+C\left(\sigma_{\varepsilon} \varepsilon^{-1}\right)^{p}\|\mathcal{Z}\|^{p}+C\left\|e^{\varepsilon^{-2} T \mathcal{A}}\right\|^{p} .
$$

By Lemmas 17 and 18, we obtain, for $\kappa_{0} \leq \kappa$,

$$
\begin{aligned}
\mathbb{E} \sup _{\left[0, \tau^{*}\right]}\|\psi\|_{\alpha}^{p} & \leq C+C\left(\sigma_{\varepsilon} \varepsilon^{-1}\right)^{p} \varepsilon^{-\kappa_{0}}+C \varepsilon^{p-3 \kappa p} \\
& \leq \varepsilon^{-\kappa_{0}}\left[C+C \sigma_{\varepsilon} \varepsilon^{-1}+C \varepsilon^{1-3 \kappa}\right]^{p}
\end{aligned}
$$

If $\sigma_{\varepsilon}=\varepsilon\left(\right.$ or $\left.\sigma_{\varepsilon}=\varepsilon^{\frac{3}{2}}\right)$, then as $\kappa<\frac{1}{3}$ we obtain (29).

Let us now state a result similar to the averaging, but where we integrate over the contribution of the initial condition in $\psi$. This always leads to terms of order $\mathcal{O}\left(\varepsilon^{2}\right)$.

Lemma 20 If Assumption 1 hold and $\psi(0)=\mathcal{O}(1)$, then for $q \geq 1$ there exist a constant $C>0$ such that

$$
\sup _{T \geq 0} \int_{0}^{T}\left\|e^{\tau \varepsilon^{-2} \mathcal{A}_{s}} \psi(0)\right\|_{\alpha}^{q} d \tau \leq C \varepsilon^{2}
$$

Proof. Using (2) we obtain

$$
\int_{0}^{T}\left\|e^{\varepsilon^{-2} \mathcal{A}_{s} \tau} \psi(0)\right\|_{\alpha}^{q} d \tau \leq c \int_{0}^{T} e^{-q \varepsilon^{-2} \omega \tau}\|\psi(0)\|_{\alpha}^{q} d \tau \leq \frac{\varepsilon^{2}}{q \omega}\|\psi(0)\|_{\alpha}^{q} .
$$




\section{Proof of the Approximation Theorem I}

This section is devoted to prove the Theorem 13 for the approximation given (17) of the solution $u$ of the SPDE (1). Before we prove our Theorem 13, let us state without proof the averaging Lemma 5.1 from [4] over the fast OU process $\mathcal{Z}_{k}$. This lemma show that the integrals over the OU-process containing odd powers like $\mathcal{Z}_{k}$ or $\mathcal{Z}_{k}^{3}$, are small. Even mixed powers like $\mathcal{Z}_{k} \mathcal{Z}_{\ell}$ or $\mathcal{Z}_{k}^{2} \mathcal{Z}_{\ell}$ do not contribute. Only even powers like $\mathcal{Z}_{k}^{2}, \mathcal{Z}_{k}^{4}$, and $\mathcal{Z}_{k}^{2} \mathcal{Z}_{\ell}^{2}$ have a contribution, which is a constant of order one.

Lemma 21 For all $\varepsilon$ let $X$ be a real valued stochastic process such that for some $r \geq 0$ we have $X(0)=\mathcal{O}\left(\varepsilon^{-r}\right)$ and $d X=G d T$ with $G=\mathcal{O}\left(\varepsilon^{-r}\right)$.

Fix any $\kappa_{0}>0$. Then for any nonnegative integers $n_{1}, n_{2}, n_{3}$ not all zero and for all triples of different indices $k_{1}, k_{2}, k_{3} \in \mathbb{N}$, we obtain

$$
\begin{aligned}
\int_{0}^{T} X \mathcal{Z}_{k_{1}}^{n_{1}} \mathcal{Z}_{k_{2}}^{n_{2}} \mathcal{Z}_{k_{3}}^{n_{3}} d \tau= & \sum_{i=1}^{3} \frac{n_{i}\left(n_{i}-1\right) \alpha_{k_{i}}^{2}}{2\left(n_{1} \lambda_{k_{1}}+n_{2} \lambda_{k_{2}}+n_{3} \lambda_{k_{3}}\right)} \int_{0}^{T} X \mathcal{Z}_{k_{1}}^{n_{1}} \mathcal{Z}_{k_{2}}^{n_{2}} \mathcal{Z}_{k_{3}}^{n_{3}} \mathcal{Z}_{k_{i}}^{-2} d \tau \\
& +\mathcal{O}\left(\varepsilon^{1-r-\left(n_{1}+n_{2}+n_{3}\right) \kappa_{0}}\right)
\end{aligned}
$$

where the fast OU-process $\mathcal{Z}_{k}$ is defined in (18).

Note that in the previous Lemma $X$ might depend on $\varepsilon$. Especially in the applications $G$ will depend on $\varepsilon$. The dependence of $X(0)$ on $\varepsilon$ does not change the result. Thus here we allow for $\varepsilon$-dependent initial conditions of order $\mathcal{O}\left(\varepsilon^{-r}\right)$.

Lemma 22 If Assumptions 1, 2, 3, 5 and 6 with only the mode $k$ forced hold and $\psi(0)=\mathcal{O}(1)$, then

$$
\begin{aligned}
a(T)= & a(0)+\int_{0}^{T} \mathcal{L}_{c} a(\tau)+\tilde{F}(a(\tau))+\tilde{G} a(\tau) d \tau \\
& +\frac{2 \alpha_{k}}{\lambda_{k}} \int_{0}^{T} B_{c}\left(a, e_{k}\right) d \tilde{\beta}_{k}+R(T),
\end{aligned}
$$

where

$$
R=\mathcal{O}\left(\varepsilon^{1-12 \kappa}\right),
$$

for $\kappa>0$ from the definition of $\tau^{*}$.

Proof. From the mild solution of equation (10) and Lemma 17 we obtain (recall that in this case $\mathcal{Z}=Z_{k} e_{k}$ )

$$
\psi(T)=y_{\varepsilon}(T)+\mathcal{Z}(T)+\mathcal{O}\left(\varepsilon^{1-3 \kappa}\right),
$$

where

$$
y_{\varepsilon}(T)=e^{\varepsilon^{-2} T \mathcal{A}_{s}} \psi(0) .
$$


Substituting from (33) into (14) and using Assumptions 3 and 5 to obtain

$$
\begin{aligned}
a(T)= & a(0)+\int_{0}^{T}\left[\mathcal{L}_{c} a+\tilde{F}(a)-2 B_{c}\left(B_{c}(\mathcal{Z}, \mathcal{Z}), \mathcal{A}_{s}^{-1} \mathcal{Z}\right)\right] d \tau \\
& -\int_{0}^{T}\left[4 \mathcal{Z}_{k}^{2} B_{c}\left(B_{c}\left(a, e_{k}\right), \mathcal{A}_{s}^{-1} e_{k}\right)+4 \mathcal{Z}_{k} B_{c}\left(a, \mathcal{A}_{s}^{-1} B_{s}\left(a, e_{k}\right)\right)\right] d \tau \\
& -\int_{0}^{T}\left[2 \mathcal{Z}_{k}^{2} B_{c}\left(a, \mathcal{A}_{s}^{-1} B_{s}\left(e_{k}, e_{k}\right)\right)-3 \mathcal{Z}_{k} \mathcal{F}_{c}\left(a, a, e_{k}\right)\right] d \tau \\
& +\int_{0}^{T}\left[3 \mathcal{Z}_{k}^{2} \mathcal{F}_{c}\left(a, e_{k}, e_{k}\right)+\mathcal{Z}_{k}^{3} \mathcal{F}_{c}\left(e_{k}\right)\right] d \tau-2 \int_{0}^{T} B_{c}\left(a, \mathcal{A}_{s}^{-1} e_{k}\right) d \beta_{k} \\
& +\sum_{\ell \neq k} \frac{2 B_{c}\left(e_{k}, e_{\ell}\right)}{\left(\lambda_{k}+\lambda_{\ell}\right)} \int_{0}^{T} 2 \mathcal{Z}_{k}^{2} B_{\ell}\left(a, e_{k}\right) d \tau+R_{1},
\end{aligned}
$$

where we do not state the lengthy expression for $R_{1}$ explicitly, but using Lemma 20 it is straightforward to prove that

$$
R_{1}=\mathcal{O}\left(\varepsilon^{1-12 \kappa}\right) .
$$

Applying finally Lemma 21 to (34), yields (31).

Lemma 23 Let Assumptions 1, 2, 3, 5 and 7 , hold. Define $b(t)$ in $\mathcal{N}$ as the solution of (15). If the initial condition satisfies $\mathbb{E}|b(0)|^{4 p} \leq C$ for some $p>1$, then there exists another constant $C$ such that

$$
\mathbb{E} \sup _{T \in\left[0, T_{0}\right]}|b(T)|^{2 p} \leq C .
$$

Proof. Applying Itô's formula we first see that $|b(T)|^{2}$ is a real-valued diffusion process. Thus again by Itô's formula applied to $\left(|b(T)|^{2}\right)^{p}$ we derive

$$
\begin{aligned}
|b(T)|^{2 p}= & |b(0)|^{2 p}+2 p \int_{0}^{T}|b(s)|^{2(p-1)}\langle b(s), d b(s)\rangle \\
& +p \int_{0}^{T}|b(s)|^{2(p-1)}\langle d b(s), d b(s)\rangle \\
& +2 p(p-1) \int_{0}^{T}|b(s)|^{2(p-2)}\langle b(s), d b(s)\rangle^{2} .
\end{aligned}
$$

From (15) we have

$$
\begin{aligned}
|b(T)|^{2 p}= & |b(0)|^{2 p}+2 p \int_{0}^{T}|b(s)|^{2 p-2}\left\langle b(s), \mathcal{L}_{c} b(s)+\tilde{F}(b(s))+\tilde{G} b(s)\right\rangle d s \\
& +C_{4} \int_{0}^{T}|b(s)|^{2 p-2}\left\langle b, B_{c}\left(b, e_{k}\right)\right\rangle d \tilde{\beta}_{k}
\end{aligned}
$$




$$
\begin{aligned}
& +C_{5} \int_{0}^{T}|b(s)|^{2 p-2}\left\langle B_{c}\left(b, e_{k}\right), B_{c}\left(b, e_{k}\right)\right\rangle d s \\
& +C_{6} \int_{0}^{T}|b(s)|^{2 p-4}\left\langle b, B_{c}\left(b, e_{k}\right)\right\rangle^{2} d s .
\end{aligned}
$$

Using Cauchy-Schwarz inequality and Assumption 5, together with Corollary 8 or Assumption 7, we obtain $(c \geq 0)$

$$
\begin{aligned}
|b(T)|^{2 p} & =|b(0)|^{2 p}+C \int_{0}^{T}|b(s)|^{2 p} d s-c \int_{0}^{T}|b(s)|^{2 p+2} d s \\
& +C_{4} \int_{0}^{T}|b(s)|^{2 p-2}\left\langle b, B_{c}\left(b, e_{k}\right)\right\rangle d \tilde{\beta}_{k} .
\end{aligned}
$$

Taking the expectations on both sides, yields

$$
\mathbb{E}|b(T)|^{2 p} \leq \mathbb{E}|b(0)|^{2 p}+C \int_{0}^{T} \mathbb{E}|b(s)|^{2 p} d s,
$$

where we used we used that stochastic integrals have 0 expectation. Applying now Gronwall's lemma to obtain

$$
\sup _{T \in\left[0, T_{0}\right]} \mathbb{E}|b(T)|^{2 p} \leq C .
$$

With $2 p$ instead of $p$ we have

$$
\sup _{T \in\left[0, T_{0}\right]} \mathbb{E}|b(T)|^{4 p} \leq C .
$$

Taking expectation after supremum on both sides of (37)

$$
\begin{aligned}
\mathbb{E} \sup _{T \in\left[0, T_{0}\right]}|b(T)|^{2 p} \leq & \mathbb{E}|b(0)|^{2 p}+C \mathbb{E} \sup _{T \in\left[0, T_{0}\right]} \int_{0}^{T}|b(s)|^{2 p} d s \\
& +C_{4} \mathbb{E} \sup _{T \in\left[0, T_{0}\right]} \int_{0}^{T}|b(s)|^{2 p-2}\left\langle b, B_{c}\left(b, e_{k}\right)\right\rangle d \tilde{\beta}_{k} .
\end{aligned}
$$

Using Burkholder-Davis-Gundy inequality (cf. [10] or [11])

$$
\mathbb{E} \sup _{T \in\left[0, T_{0}\right]}|b(T)|^{2 p} \leq \mathbb{E}|b(0)|^{2 p}+C \int_{0}^{T_{0}} \mathbb{E}|b(s)|^{2 p} d s+C \mathbb{E}\left(\int_{0}^{T_{0}}|b(s)|^{4 p} d s\right)^{1 / 2} .
$$

Using our first bounds on $b$ from (38) and (39) after Hölder, yields (36).

Theorem 24 Assume that Assumptions 1, 2, 3, 5, 6 and 7 hold, and suppose $a(0)=\mathcal{O}(1)$ and $\psi(0)=\mathcal{O}(1)$. Let $b$ be a solution of (15) and $a$ as defined in (8). If the initial conditions satisfy $a(0)=b(0)$, then for $\kappa<\frac{1}{12}$ we obtain

$$
\mathbb{E} \sup _{T \in\left[0, \tau^{*}\right]}\|a(T)-b(T)\|^{2 p} \leq C \varepsilon^{2 p(1-12 \kappa)} .
$$


Proof. Subtracting (15) from (31) and defining $h:=a-b$, we obtain

$$
\begin{aligned}
h(T)=\int_{0}^{T}\left[\mathcal{L}_{c} h+\tilde{F}\right. & (h+b)-\tilde{F}(b)+\tilde{G} h] d \tau \\
& +\int_{0}^{T} \frac{2 \alpha_{k}}{\lambda_{k}} B_{c}\left(h, e_{k}\right) d \tilde{\beta}_{k}+R(T) .
\end{aligned}
$$

In order to apply Ito-formula, we define $\tilde{h}=h-R$. Thus,

$$
d \tilde{h}=\left[\mathcal{L}_{c} h+\tilde{F}(h+b)-\tilde{F}(b)+\tilde{G} h\right] d T+\frac{2 \alpha_{k}}{\lambda_{k}} B_{c}\left(h, e_{k}\right) d \tilde{\beta}_{k} .
$$

Now Itô-formula yields

$$
\frac{1}{2 p} d|\tilde{h}|^{2 p}=|\tilde{h}|^{2 p-2}\langle\tilde{h}, d \tilde{h}\rangle+|\tilde{h}|^{2 p-2}\langle d \tilde{h}, d \tilde{h}\rangle+|\tilde{h}|^{2 p-4}\langle\tilde{h}, d \tilde{h}\rangle^{2} .
$$

For the nonlinear term we use Assumption 7:

$$
\langle\tilde{F}(\tilde{h}+b+R)-\tilde{F}(b), \tilde{h}\rangle \leq-c|\tilde{h}|^{4}+C|R|^{4}+C|R|^{2}|b|^{2} .
$$

for some $c \geq 0$. Thus (note that we keep some of the $h=\tilde{h}+R$ )

$$
\begin{aligned}
& \frac{1}{2 p} d|\tilde{h}|^{2 p}+c|\tilde{h}|^{2 p+2} d T-|\tilde{h}|^{2 p-2}\left\langle\tilde{h}, \frac{2 \alpha_{k}}{\lambda_{k}} B_{c}\left(h, e_{k}\right) d \tilde{\beta}_{k}\right\rangle \\
& \leq C|\tilde{h}|^{2 p-2}\left[|R|^{4}+|R|^{2}|b|^{2}\right] d T+C|\tilde{h}|^{2 p-2}|h|^{2} d T+C|\tilde{h}|^{2 p-4}|h|^{4} d T \\
& \leq C|\tilde{h}|^{2 p-2}\left[|R|^{4}+|R|^{2}+|R|^{2}|b|^{2}\right] d T+C|\tilde{h}|^{2 p} d T+C|\tilde{h}|^{2 p-4}|R|^{4} d T \\
& \leq C|\tilde{h}|^{2 p} d T+C\left[|R|^{4 p}+|R|^{2 p}+|R|^{2 p}|b|^{2 p}\right] d T,
\end{aligned}
$$

where we used Young's inequality frequently. For example, $x^{2 p-\ell} y^{\ell} \leq C x^{2 p}+$ $C y^{2 p}$. Now consider for an appropriate constant $C_{0}>0$

$$
\begin{aligned}
d\left[e^{-C_{0} T}|\tilde{h}(T)|^{2 p}\right] & +2 p c e^{-C_{0} T}|\tilde{h}|^{2 p+2} d T-2 p e^{-C_{0} T}|\tilde{h}|^{2 p-2}\left\langle\tilde{h}, \frac{2 \alpha_{k}}{\lambda_{k}} B_{c}\left(h, e_{k}\right) d \tilde{\beta}_{k}\right\rangle \\
& \leq C e^{-C_{0} T}\left[|R(T)|^{4 p}+|R(T)|^{2 p}+|R(T)|^{2 p}|b(T)|^{2 p}\right] d T
\end{aligned}
$$

Integration up to $T \wedge \tau^{*}$ and taking expectation (recall that $\tilde{h}(0)=R(0)=0$ )

$$
\begin{aligned}
\mathbb{E}\left|\tilde{h}\left(T \wedge \tau^{*}\right)\right|^{2 p} & \leq \mathbb{E} \int_{0}^{T \wedge \tau^{*}}\left[|R|^{4 p}+|R|^{2 p}+|R|^{2 p}|b|^{2 p}\right] d s \\
& \leq C \varepsilon^{(1-12 \kappa) 2 p}
\end{aligned}
$$

where we used Hölder, $\tau^{*} \leq T_{0}$, together with Lemma 23 and 22 .

Taking a step back, recall that we had for $T \leq \tau^{*}$

$$
\begin{aligned}
\frac{1}{2 p}|\tilde{h}(T)|^{2 p} & \leq \int_{0}^{T}|\tilde{h}|^{2 p-2}\left\langle\tilde{h}, \frac{2 \alpha_{k}}{\lambda_{k}} B_{c}\left(h, e_{k}\right) d \tilde{\beta}_{k}\right\rangle \\
& +C \int_{0}^{T}|\tilde{h}|^{2 p} d s+C \int_{0}^{T}\left[|R|^{4 p}+|R|^{2 p}+|R|^{2 p}|b|^{2 p}\right] d s
\end{aligned}
$$


In order to avoid problems with $\tilde{h}$ being defined only up to the stopping time $\tau^{*}$, we define the stopped process $\tilde{h}_{\tau^{*}}(s)=\tilde{h}\left(s \wedge \tau^{*}\right)$. Thus

$$
\begin{aligned}
\frac{1}{2 p} \sup _{T \in\left[0, \tau^{*}\right]}|\tilde{h}(T)|^{2 p} & \leq \sup _{T \in\left[0, \tau^{*}\right]} \int_{0}^{T}\left|\tilde{h}_{\tau^{*}}\right|^{2 p-2}\left\langle\tilde{h}_{\tau^{*}}, \frac{2 \alpha_{k}}{\lambda_{k}} B_{c}\left(\tilde{h}_{\tau^{*}}, e_{k}\right) d \tilde{\beta}_{k}\right\rangle \\
& +\sup _{T \in\left[0, \tau^{*}\right]} \int_{0}^{T}\left|\tilde{h}_{\tau^{*}}\right|^{2 p-2}\left\langle\tilde{h}_{\tau^{*}}, \frac{2 \alpha_{k}}{\lambda_{k}} B_{c}\left(\tilde{h}_{\tau^{*}}, e_{k}\right) d \tilde{\beta}_{k}\right\rangle \\
& +C \int_{0}^{\tau^{*}}\left|\tilde{h}_{\tau^{*}}\right|^{2 p} d s+C \int_{0}^{\tau^{*}}\left[|R|^{4 p}+|R|^{2 p}+|R|^{2 p}|b|^{2 p}\right] d s
\end{aligned}
$$

Now we can take suprema up to $T_{0}$ on the right hand side. Taking now expectation, Burkholder-Davis-Gundy together with (32) and (41) yields

$$
\mathbb{E} \sup _{T \in\left[0, \tau^{*}\right]}|\tilde{h}(T)|^{2 p} \leq C \varepsilon^{(1-12 \kappa) 2 p}
$$

Finally, using

$$
\mathbb{E} \sup _{\left[0, \tau^{*}\right]}|a-b|^{2 p}=\mathbb{E} \sup _{\left[0, \tau^{*}\right]}|h|^{2 p} \leq C \mathbb{E} \sup _{\left[0, \tau^{*}\right]}|\tilde{h}|^{2 p}+C \mathbb{E} \sup _{\left[0, \tau^{*}\right]}|R|^{2 p}
$$

yields the result together with Lemma 22 and (42).

Now, we can use the results obtained in the previous proofs to verify the main result of Theorem 13.

Proof of Theorem 13. First we show that $\tau^{*}=T_{0}$ with high probability. Note that

$$
\begin{aligned}
\Omega & \supset\left\{\tau^{*}=T_{0}\right\} \\
& \supset\left\{\sup _{\left[0, T_{0}\right]}\left\|u\left(T \varepsilon^{-2}\right)\right\|_{\alpha}<\frac{1}{2} \varepsilon^{1-\kappa}\right\} \\
& \supset\left\{\sup _{\left[0, T_{0}\right]}\|a\|_{\alpha}<\frac{1}{4} \varepsilon^{-\kappa}, \sup _{\left[0, T_{0}\right]}\|\psi\|_{\alpha}<\frac{1}{4} \varepsilon^{-\kappa}\right\} \\
& \supset\left\{\sup _{\left[0, T_{0}\right]}\|a-b\|_{\alpha}<\frac{1}{8} \varepsilon^{-\kappa}, \sup _{\left[0, T_{0}\right]}\|b\|_{\alpha}<\frac{1}{8} \varepsilon^{-\kappa}, \sup _{\left[0, T_{0}\right]}\|\psi\|_{\alpha}<\frac{1}{4} \varepsilon^{-\kappa}\right\} \\
& =: \Omega_{\varepsilon} .
\end{aligned}
$$

Hence

$$
\begin{aligned}
\mathbb{P}\left\{\tau^{*}<T_{0}\right\} & =1-\mathbb{P}\left\{\tau^{*}=T_{0}\right\} \leq 1-\mathbb{P}\left\{\Omega_{\varepsilon}\right\}=\mathbb{P}\left\{\left(\Omega_{\varepsilon}\right)^{c}\right\} \\
& \leq \mathbb{P}\left\{\sup _{\left[0, T_{0}\right]}\|a-b\|_{\alpha} \geq \frac{1}{8} \varepsilon^{-\kappa}\right\}+\mathbb{P}\left\{\sup _{\left[0, T_{0}\right]}\|b\|_{\alpha} \geq \frac{1}{8} \varepsilon^{-\kappa}\right\} \\
& \quad+\mathbb{P}\left\{\sup _{\left[0, T_{0}\right]}\|\psi\|_{\alpha} \geq \frac{1}{4} \varepsilon^{-\kappa}\right\} \\
& \leq C \varepsilon^{(1-11 \kappa) p}+C \varepsilon^{\kappa p}+C \varepsilon^{\left(\kappa-\kappa_{0}\right) p},
\end{aligned}
$$


where we used Chebychev's inequality together with Theorems 24, 23, and Corollary 19. If we choose $\kappa<\frac{1}{12}$ and $\kappa_{0}$ from Corollary 19 sufficiently small, we obtain that for all $p>1$ there is a constant such that

$$
\mathbb{P}\left\{\tau^{*}<T_{0}\right\} \leq C \varepsilon^{p} .
$$

Now let us turn to the approximation result. Using Theorem 24 and Lemma 17 , yields

$$
\begin{aligned}
& \mathbb{E} \sup _{T \in\left[0, \tau^{*}\right]}\left\|u\left(\varepsilon^{-2} T\right)-\varepsilon b(T)-\varepsilon \mathcal{Q}(T)\right\|_{\alpha}^{p} \\
& \leq C \varepsilon \mathbb{E} \sup _{\left[0, \tau^{*}\right]}\|a-b\|_{\alpha}^{p}+C \varepsilon \mathbb{E} \sup _{\left[0, \tau^{*}\right]}\|\psi-\mathcal{Q}\|_{\alpha}^{p} \\
& \leq C \varepsilon^{p(2-12 \kappa)}+C \varepsilon^{p(2-3 \kappa)} \leq C \varepsilon^{p(2-12 \kappa)} .
\end{aligned}
$$

Thus

$$
\begin{aligned}
& \mathbb{P}\left(\sup _{t \in\left[0, \varepsilon^{-2} T_{0}\right]}\left\|u(t)-\varepsilon b\left(\varepsilon^{2} t\right)-\varepsilon \mathcal{Q}\left(\varepsilon^{2} t\right)\right\|_{\alpha}>\varepsilon^{2-13 \kappa}\right) \\
& =\mathbb{P}\left(\sup _{t \in\left[0, \varepsilon^{-2} T_{0}\right]}\left\|u(t)-\varepsilon b\left(\varepsilon^{2} t\right)-\varepsilon \mathcal{Q}\left(\varepsilon^{2} t\right)\right\|_{\alpha}>\varepsilon^{2-13 \kappa}, \tau^{*}=T_{0}\right) \\
& \quad+\mathbb{P}\left(\sup _{t \in\left[0, \varepsilon^{-2} T_{0}\right]}\left\|u(t)-\varepsilon b\left(\varepsilon^{2} t\right)-\varepsilon \mathcal{Q}\left(\varepsilon^{2} t\right)\right\|_{\alpha}>\varepsilon^{2-13 \kappa}, \tau^{*}<T_{0}\right) \\
& \quad \leq \mathbb{P}\left(\sup _{t \in\left[0, \varepsilon^{-2} \tau^{*}\right]}\left\|u(t)-\varepsilon b\left(\varepsilon^{2} t\right)-\varepsilon \mathcal{Q}\left(\varepsilon^{2} t\right)\right\|_{\alpha}>\varepsilon^{2-13 \kappa}\right)+\mathbb{P}\left(\tau^{*}<T_{0}\right) \\
& \leq C \varepsilon^{-q(2-13 \kappa)} \varepsilon^{q(2-12 \kappa)}+C \varepsilon^{q} \\
& \leq C \varepsilon^{q \kappa},
\end{aligned}
$$

where we used again Chebychev's inequality and (45) and (46). If $p=q \kappa$ we obtain our final result (19).

\subsection{Application of Approximation Theorem I}

In the literature there are numerous examples of equations with quadratic nonlinearities (Burgers' equation) or with cubic nonlinearities (GinzburgLandau / Allen-Cahn equation) or both (Swift-Hohenberg equation) where our theory does apply.

\subsubsection{Burgers' Equation}

The first example is the stochastic Burgers' equation already studied in [2]

$$
\partial_{t} u=-\left(\partial_{x}^{2}+1\right) u+\nu \varepsilon^{2} u-u \partial_{x} u+\varepsilon \partial_{t} W(t),
$$

on the interval $[0, \pi]$, with Dirichlet boundary conditions. We take

$$
\mathcal{H}=L^{2}([0, \pi]), \quad e_{k}(x)=\sqrt{\frac{2}{\pi}} \sin (k x) \quad \text { and } \mathcal{N}=\operatorname{span}\{\sin \} .
$$


Assumption 1 is true with $m=2$ and $\lim _{k \rightarrow \infty} \lambda_{k}=\infty$, where the eigenvalues of $-\mathcal{A}=-\partial_{x}^{2}-1$ are $\lambda_{k}=k^{2}-1$. If we fix $P_{c}$ to be the $\mathcal{H}$-orthogonal projection onto $\mathcal{N}$, then both $P_{c}$ and $P_{s}$ commute with $\mathcal{A}$.

Moreover, all conditions of Assumption 3 are satisfied for the operator

$$
B(u, v)=\frac{1}{2} \partial_{x}(u v),
$$

as follows:

$$
P_{c} B(u, u)=P_{c}\left[\gamma^{2} \sin (x) \cos (x)\right]=0 \text { for } u=\gamma \sin \in \mathcal{N},
$$

and for $\alpha=\frac{1}{4}$ and $\beta=\frac{5}{4}<m$ we obtain

$$
2\|B(u, v)\|_{\mathcal{H}^{-1}}=\left\|\partial_{x}(u v)\right\|_{\mathcal{H}^{-1}} \leq\|u v\|_{L^{2}} \leq C\|u\|_{\mathcal{H}^{\frac{1}{4}}}\|v\|_{\mathcal{H}^{\frac{1}{4}}},
$$

where we used Sobolev embedding of $\mathcal{H}^{1 / 4}$ into $L^{4}$. If the noise acts on the second mode (i.e., $W(t, x)=\sigma \beta_{2}(t) \sin (2 x)$ ), then our main theorem states that for

$$
u(t)=\varepsilon v\left(\varepsilon^{2} t\right)
$$

we have

$$
v(T) \simeq b(T) \sin +\varepsilon \frac{\sigma}{3} \partial_{T} \tilde{\beta}_{2}(T) \sin (2 x),
$$

where $b$ is the solution of the amplitude equation of Stratonovic type

$$
d b=\left[\left(\nu-\frac{\sigma^{2}}{88}\right) b-\frac{1}{12} b^{3}\right] d T-\frac{\sigma}{6} b \circ d \tilde{\beta}_{2} .
$$

If $\sigma^{2}>88 \nu$, then $\nu-\frac{\sigma^{2}}{88}$ is negative. In this case sufficiently strong degenerate additive noise stabilizes the dynamics of the dominant modes.

\subsubsection{Ginzburg-Landau / Allen-Cahn equation}

The second example is the stochastic Ginzburg-Landau / Allen-Cahn equation

$$
\partial_{t} u=\left(\partial_{x}^{2}+1\right) u+\nu \varepsilon^{2} u-u^{3}+\varepsilon \partial_{t} W(t),
$$

subject to Dirichlet boundary conditions on the interval $[0, \pi]$. We note that

$$
\mathcal{A}=\partial_{x}^{2}+1, \quad \mathcal{L}=\nu \mathcal{I}, \mathcal{F}(u)=-u^{3} .
$$

If we take $\mathcal{H}, e_{k}$, and $\mathcal{N}$ as in the previous example, then Assumption 1 is again true. Moreover, it is easy to check that the condition (3) on the nonlinearity is satisfied for $\alpha=1$ and $\beta=0$. For the Assumption 6 on the Wiener process, we consider here noise acting only on $\sin (2 x)$.

The main theorem states that the solution $u(t)=\varepsilon v\left(\varepsilon^{2} t\right)$ of (47) is approximated by

$$
v(T) \simeq b(T) \sin +\varepsilon \frac{\sigma}{3} \partial_{T} \tilde{\beta}_{2}(T) \sin (2 x)
$$


where $b$ is the solution of the amplitude equation takes the form

$$
b^{\prime}=\left(\nu-\frac{\sigma^{2}}{4}\right) b-\frac{3}{4} b^{3},
$$

where $\mathcal{F}_{c}\left(u, e_{k}, e_{k}\right)=-\frac{1}{\pi} u$. Note that here the Amplitude equation is deterministic, and we have a stabilization of the dominant modes provided $\sigma^{2}>4 \nu$. Here too much noise destroys the dominant pattern.

\subsubsection{Generalized Swift-Hohenberg equation}

The Swift-Hohenberg equation was defined in introduction (cf. (21)). It has been used as a toy model for the convective instability in Rayleigh-Bénard problem (see [7] or [16]). Now it is one of the celebrated models in the theory of pattern formation. For this model we note that

$$
\mathcal{A}=-\left(1+\partial_{x}^{2}\right)^{2}, \quad \mathcal{L}=\nu \mathcal{I}, \mathcal{F}(u)=-u^{3}, \quad B(u, u)=\gamma u^{2} .
$$

If we take

$$
e_{k}(x)= \begin{cases}\frac{1}{\sqrt{\pi}} \sin (k x) & \text { if } k>0, \\ \frac{1}{\sqrt{2 \pi}} & \text { if } k=0, \\ \frac{1}{\sqrt{\pi}} \cos (k x) & \text { if } k<0,\end{cases}
$$

and

$$
\mathcal{H}=L^{2}([0,2 \pi]) \text { and } \mathcal{N}=\operatorname{span}\{\sin , \cos \},
$$

then the eigenvalues of $-\mathcal{A}=\left(1+\partial_{x}^{2}\right)^{2}$ are $\lambda_{k}=\left(1-k^{2}\right)^{2}$ for $k \in \mathbb{N}_{0}$ with $m=4, \lambda_{0}=1>0$ and $\lim _{k \rightarrow \infty} \lambda_{k}=\infty$. Moreover, with $\alpha=1$ and $\beta=0$, it is easy to check that

$$
\|\mathcal{F}(u, v, w)\|_{\mathcal{H}^{1}}=\|-u v w\|_{\mathcal{H}^{1}} \leq C\|u\|_{\mathcal{H}^{1}}\|v\|_{\mathcal{H}^{1}}\|w\|_{\mathcal{H}^{1}} .
$$

For Assumption 6, we consider two cases:

First case. The noise is a constant in the space (i.e. $W(t)=\frac{\alpha_{0}}{\sqrt{2 \pi}} \beta_{0}(t)=$ $\left.\sigma_{0} \beta_{0}(t)\right)$. In this case our main theorem states that the solution $u(t, x)=$ $\varepsilon v\left(\varepsilon^{2} t, x\right)$, of (21) approximated by

$$
v(T, x) \simeq b_{1}(T) \sin (x)+b_{-1}(T) \cos (x)+\varepsilon \sigma_{0} \partial_{T} \tilde{\beta}_{0}(T)+\mathcal{O}\left(\varepsilon^{1-}\right),
$$

where $b_{1}$ and $b_{-1}$ are the solution of the amplitude equation

$d b_{i}=\left[\left(\nu-\frac{3}{2} \sigma_{0}^{2}+3 \sigma_{0}^{2} \gamma^{2}\right) b_{i}+\frac{3}{4}\left(\frac{38}{27} \gamma^{2}-1\right) b_{i}\left(b_{1}^{2}+b_{-1}^{2}\right)\right] d T+2 \gamma \sigma_{0} b_{i} d \tilde{\beta}_{0}$ for $i= \pm 1$.

Second case. If the noise acts on $\sin (2 x)($ or $\cos (2 x))$, then the amplitude equations are

$d b_{i}^{\prime}=\left[\left(\nu-\frac{1}{12} \sigma_{2}^{2}+\frac{1}{16} \sigma_{2}^{2} \gamma^{2}\right) b_{i}+\frac{3}{4}\left(\frac{38}{27} \gamma^{2}-1\right) b_{i}\left(b_{1}^{2}+b_{-1}^{2}\right)\right] d T+\frac{\gamma \sigma_{2}}{9} b_{-i} d \tilde{\beta}_{2}$ for $i= \pm 1$, 
where $\sigma_{2}=\frac{\alpha_{2}}{\sqrt{\pi}}$.

Here the question of stabilization is not obvious to decide. In both cases we need $\gamma^{2}<27 / 38$ in order to have a stable cubic, and to apply our main result. After using the Ito-Stratonovic-correction, we have that for $\gamma^{2}<2 / 3$ in the first case and $\gamma^{2}<1.46$ in the second case, large noise will stabilize.

\section{Proof of the Approximation Theorem II}

In this section, we use many lemmas and ideas of the previous sections, as the main ideas are similar.

Lemma 25 If Assumptions 1, 2, 4, 5 and 6 hold, together with $\psi(0)=$ $\mathcal{O}(1)$, then

$$
a(T)=a(0)+\int_{0}^{T}\left[\mathcal{L}_{c} a+\tilde{F}(a)\right] d \tau+\frac{1}{4} \sum_{k} \frac{\alpha_{k}^{2}}{\lambda_{k}} B_{c}\left(e_{k}, e_{k}\right) T+\mathcal{O}\left(\varepsilon^{1 / 2-9 \kappa}\right),
$$

for $\frac{1}{6}>\kappa>0$ from the definition of $\tau^{*}$.

Proof. From the mild solution of equation (10) and Lemma 17, with $\sigma_{\varepsilon}=\varepsilon^{3 / 2}$, we obtain

$$
\begin{aligned}
\psi(T) & =y_{\varepsilon}(T)+\varepsilon^{1 / 2} \mathcal{Z}(T)+\mathcal{O}\left(\varepsilon^{1-3 \kappa}\right) \\
& =y_{\varepsilon}(T)+\mathcal{O}\left(\varepsilon^{1 / 2-3 \kappa}\right)
\end{aligned}
$$

where as before

$$
y_{\varepsilon}(T)=e^{\varepsilon^{-2} T \mathcal{A}_{s}} \psi(0) .
$$

Substituting from (50) into (14) and using Assumptions 4, 5 and using Burkholder-Davis-Gundy inequality to obtain (49). Here we follow the lines of the proof of our first theorem. The proof is a straightforward modification.

Lemma 26 Let Assumptions 1, 2, 4 and 5, hold. Define $b(t)$ in $\mathcal{N}$ as the solution of (22). If the initial condition satisfies $\mathbb{E}|b(0)|^{2 p} \leq C$ for some $p \geq 1$, then there exists a constant $C>0$ such that

$$
\mathbb{E} \sup _{T \in\left[0, T_{0}\right]}|b(T)|^{2 p} \leq C .
$$

Proof. Taking the scalar product $\langle b, \cdot\rangle$ on both sides of $(22)$, and using Corollary 8, yields

$$
\begin{aligned}
\frac{1}{2} \partial_{T}|b|^{2} & \leq\left\langle b, \mathcal{L}_{c} b+\tilde{F}(b)+\sum_{k} \frac{\alpha_{k}^{2}}{4 \lambda_{k}} B_{c}\left(e_{k}, e_{k}\right)\right\rangle \\
& \leq C|b|^{2}+C_{1}
\end{aligned}
$$


Using Gronwall's lemma we obtain for $0 \leq T$ that

$$
|b(T)|^{2} \leq e^{2 C T}|b(0)|^{2}+C_{1} e^{2 C T} .
$$

We finish the proof by taking the expectation after supremum on $\left[0, T_{0}\right]$.

Theorem 27 Assume that Assumptions 1, 2, 4, 5, 6 and 7 hold and suppose $a(0)=\mathcal{O}(1)$ and $\psi(0)=\mathcal{O}(1)$. Let $b$ be a solution of the amplitude equation (22). Recall a was described in (49). If the initial conditions satisfy $a(0)=$ $b(0)$, then for $\kappa<\frac{1}{18}$ we obtain that for all $p>1$ there is a constant $C>0$ such that

$$
\mathbb{E} \sup _{T \in\left[0, \tau^{*}\right]}|a(T)-b(T)|^{p} \leq C \varepsilon^{\left(\frac{1}{2}-9 \kappa\right) p} .
$$

Proof. Subtracting (22) from (49) and defining

$$
h(T):=a(T)-b(T),
$$

we obtain

$$
h(T)=\int_{0}^{T} \mathcal{L}_{c} h(\tau) d \tau+\int_{0}^{T} \tilde{F}(h+b) d \tau-\int_{0}^{T} \tilde{F}(b) d \tau+\tilde{R}(T),
$$

where the error $\tilde{R}$ is bounded by

$$
\tilde{R}=\mathcal{O}\left(\varepsilon^{1 / 2-9 \kappa}\right)
$$

In order to apply standard techniques, we define $Q$ as

$$
Q(T):=h(T)-\tilde{R}(T) .
$$

From Equation (54) we obtain the random ODE

$$
\partial_{T} Q=\mathcal{L}_{c} Q+\mathcal{L}_{c} \tilde{R}+\tilde{F}(Q+\tilde{R}+b)-\tilde{F}(b) .
$$

Taking the scalar product $\langle Q, \cdot\rangle$ on both sides

$$
\frac{1}{2} \partial_{T}|Q(T)|=\left\langle Q, \mathcal{L}_{c} Q+\mathcal{L}_{c} \tilde{R}\right\rangle+\langle Q, \tilde{F}(Q+\tilde{R}+b)-\tilde{F}(b)\rangle .
$$

Using Young and Cauchy-Schwartz inequalities and Assumption 7, we obtain the following linear ordinary differential inequality

$$
\begin{aligned}
\frac{1}{2} \partial_{T}|Q(T)|^{2} & \leq C|Q(T)|^{2}+C|\tilde{R}(T)|^{2}-c_{0}|Q(T)|^{4}+C|\tilde{R}(T)|^{2}|b(T)|^{2} \\
& \leq C|Q(T)|^{2}+C|\tilde{R}(T)|^{2}+C|\tilde{R}(T)|^{2}|b(T)|^{2}
\end{aligned}
$$

Using Gronwall's lemma, we obtain (as $Q(0)=0$ ) for $T \leq \tau^{*} \leq T_{0}$

$$
|Q(T)|^{2} \leq C \int_{0}^{T}|\tilde{R}(s)|^{2}\left[1+|b(s)|^{2}\right] e^{2 C(T-s)} d s \leq C \sup _{\left[0, \tau^{*}\right]}|\tilde{R}|^{2}\left[1+|b|^{2}\right]
$$


Taking $\frac{p}{2}$-th power, supremum, and finally the expectation yields

$$
\mathbb{E} \sup _{\left[0, \tau^{*}\right]}|Q|^{p} \leq C \varepsilon^{p / 2-9 p \kappa},
$$

where we used (51) and (55). We finish the proof by using (53), (56) and

$$
\mathbb{E} \sup _{\left[0, \tau^{*}\right]}|a-b|^{p}=\mathbb{E} \sup _{\left[0, \tau^{*}\right]}|Q+\tilde{R}|^{p} \leq \mathbb{E} \sup _{\left[0, \tau^{*}\right]}|Q|^{p}+\mathbb{E} \sup _{\left[0, \tau^{*}\right]}|\tilde{R}|^{p} .
$$

Now, we can use the results previously obtained to prove the main result of Theorem 16 for the approximation of the solution (24) of the SPDE (1).

Proof of Theorem 16. We follow the same steps of the proof of Theorem 13.

\subsection{Application of Approximation Theorem II}

Note that for Burgers equation we can not apply our approximation Theorem II because the condition $B_{c}\left(e_{k}, e_{k}\right) \neq 0$ for $k \in\{n+1, \ldots . N\}$ is not satisfied. In the case of Ginzburg-Landau equation, as this contains only cubic nonlinearities, the noise does not effect the amplitude equation. thus we do not treat this in detail here.

We apply our Theorem II to the generalized Swift-Hohenberg equation and we study different cases depending on the type of the noise.

\subsubsection{Generalized Swift-Hohenberg equation}

We study the generalized stochastic Swift-Hohenberg equation given as

$$
\partial_{t} u=-\left(4+\partial_{x}^{2}\right)^{2} u+\nu \varepsilon^{2} u+\gamma u^{2}-u^{3}+\varepsilon^{3 / 2} \partial_{t} W .
$$

For this model we note that

$$
\mathcal{A}=-\left(4+\partial_{x}^{2}\right)^{2}, \quad \mathcal{L}=\nu \mathcal{I}, \mathcal{F}(u)=-u^{3}, \quad B(u, u)=\gamma u^{2} .
$$

Later we need that $\gamma^{2}<54 / 5$. Define as the orthonormal set of eigenfunctions of $\mathcal{A}$

$$
e_{k}(x)= \begin{cases}\frac{1}{\sqrt{\pi}} \sin (k x) & \text { if } k>0, \\ \frac{1}{\sqrt{2 \pi}} & \text { if } k=0, \\ \frac{1}{\sqrt{\pi}} \cos (k x) & \text { if } k<0,\end{cases}
$$

then

$$
\mathcal{N}=\operatorname{span}\{\sin (2 x), \cos (2 x)\}, \text { and } \lambda_{k}=\left(4-k^{2}\right)^{2} .
$$

For noise, we consider three different cases, but in all cases the main theorem gives that the solution $u(t, x)=\varepsilon v\left(\varepsilon^{2} t, x\right)$ of (57) is well approximated by

$$
v(T, x)=b_{2}(T) \sin (2 x)+b_{-2}(T) \cos (2 x)+\mathcal{O}\left(\varepsilon^{1 / 2-}\right),
$$


where $b_{2}$ and $b_{-2}$ are the solution of the amplitude equation

First case. If the noise takes the form $W(t, x)=\sigma \partial_{t} \beta \cos (x)$, then

$$
\begin{aligned}
b_{2}^{\prime} & =\nu b_{2}-\left(\frac{3}{4}-\frac{5}{72} \gamma^{2}\right) b_{2}\left(b_{2}^{2}+b_{-2}^{2}\right) \\
b_{-2}^{\prime} & =\nu b_{-2}-\left(\frac{3}{4}-\frac{5}{72} \gamma^{2}\right) b_{-2}\left(b_{2}^{2}+b_{-2}^{2}\right)+\frac{\gamma \sigma^{2}}{72} .
\end{aligned}
$$

Second case. If the noise acts on $\sin (x)$, then

$$
\begin{aligned}
b_{2}^{\prime} & =\nu b_{2}-\left(\frac{3}{4}-\frac{5}{72} \gamma^{2}\right) b_{2}\left(b_{2}^{2}+b_{-2}^{2}\right) \\
b_{-2}^{\prime} & =\nu b_{-2}-\left(\frac{3}{4}-\frac{5}{72} \gamma^{2}\right) b_{-2}\left(b_{2}^{2}+b_{-2}^{2}\right)-\frac{\gamma \sigma^{2}}{72},
\end{aligned}
$$

Third case. If the noise takes the form

$$
W(t, x)=\sum_{k=-1, k \neq-2}^{-N} \alpha_{k} \partial_{t} \beta_{k} \cos (k x)+\sum_{k=1, k \neq 2}^{N} \sigma_{k} \partial_{t} \beta_{k} \sin (k x),
$$

then in this case

$$
\begin{aligned}
b_{2}^{\prime} & =\nu b_{2}-\left(\frac{3}{4}-\frac{5 \gamma^{2}}{72}\right) b_{2}\left(b_{2}^{2}+b_{-2}^{2}\right) \\
b_{-2}^{\prime} & =\nu b_{-2}-\left(\frac{3}{4}-\frac{5 \gamma^{2}}{72}\right) b_{-2}\left(b_{2}^{2}+b_{-2}^{2}\right)+\frac{\gamma}{72}\left(\alpha_{1}^{2}-\sigma_{1}^{2}\right) .
\end{aligned}
$$

We note that if we choose $\sigma_{1}= \pm \alpha_{1}$, then there is no effect of noise on the solution.

Note that in the first two cases any little contribution of noise leads to a pattern $\cos (2 x)$, as the solution of the amplitude equation is always driven out of 0 .

\section{Acknowledgement}

This work is supported by the Deutsche Forschungsgemeinschaft (DFG, "Mehrskalenanalyse stochastischer partieller Differentialgleichungen", BL535/9$2)$.

\section{References}

[1] D. Blömker and M. Hairer. Multiscale expansion of invariant measures for SPDEs. Commun. Math. Phys., 251(3):515-555, (2004).

[2] D. Blömker, M. Hairer and G. A. Pavliotis. Multiscale analysis for stochastic partial differential equations with quadratic nonlinearities. Nonlinearity, 20:1-25, (2007). 
[3] D. Blömker and W. W. Mohammed. Amplitude equations for SPDEs with quadratic nonlinearities. Electronic Journal of Probability, 14(88):2527-2550, (2009).

[4] D. Blömker and W. W. Mohammed. Amplitude equations for SPDEs with cubic nonlinearities. Stochastics. An International Journal of Probability and Stochastic Process, 85(2):181-215, (2013).

[5] D. Blömker and M. Romito, Regularity and blow up in a surface growth model. Dynamics of PDE, 6(3):227-252, (2009).

[6] J. Burke and Jonathan H. P. Dawes. Localized States in an Extended Swift-Hohenberg Equation. SIAM J. Appl. Dyn. Syst., 11(1):261-284, (2012)

[7] M. C. Cross and P. C. Hohenberg. Pattern formation outside of equilibrium, Rev. Mod. Phys. 65: 581-1112, (1993).

[8] A. Chekhlov and V. Yakhot. Kolmogorov turbulence in a random-forcedriven Burgers equation: Anomalous scaling and probability density functions. Phys. Rev. E. 52: 5681-5684, (1995)

[9] G. Da Prato and J. Zabczyk, Stochastic Equations in Infinite Dimensions, Cambridge University Press, Cambridge, UK, (1992).

[10] G. Da Prato and J. Zabczyk. Ergodicity for infinite-dimensional systems, volume 229 of London Mathematical Society Lecture Note Series. Cambridge University Press, Cambridge, (1996).

[11] E. Hausenblas and J. Seidler. A note on maximal inequality for stochastic convolutions. Czechoslovak Math. J., 51(126)(4):785-790, (2001).

[12] D. Henry, Geometric Theory of Semilinear Parabolic Equations, Lecture Notes in Mathematics 840, Springer-Verlag, Berlin, (1981).

[13] A. Hutt, A.Longtin, L.Schimansky-Geier. Additive global noise delays Turing bifurcations. Physical Review Letters, 98:230601, (2007).

[14] A. Hutt, A.Longtin, L.Schimansky-Geier. Additive noise-induced Turing transitions in spatial systems with application to neural fields and the Swift-Hohenberg equation. Physica D, Physica D 237:755-773, (2008).

[15] A. Hutt. Additive noise may change the stability of nonlinear systems. Europhys. Lett. 84, 34003, (2008).

[16] P. C. Hohenberg and J. B. Swift. Effects of additive noise at the onset of Rayleigh-Bénard convection, Physical Review A, 46:4773-4785, (1992). 
[17] K. Klepel, D. Blömker, W. W. Mohammed. Amplitude equation for the generalized Swift Hohenberg equation with noise. to appear in ZAMP - Zeitschrift fr angewandte Mathematik und Physik.

[18] A. Pazy, Semigroups of linear operators and applications to partial differential equations. Applied Mathematical Sciences, 44. New York etc.: Springer-Verlag. (1983).

[19] M. Pradas, G.A. Pavliotis, S. Kalliadasis, D.T.Papageorgiou, D. Tseluiko, Additive noise effects in active nonlinear spatially extended systems, European Journal of Applied Mathematics, 23:563-591, (2012).

[20] C. Prévôt and M. Röckner, A concise course on stochastic partial differential equations. Lecture Notes in Mathematics 1905. Berlin: Springer, (2007).

[21] A.J. Roberts. A step towards holistic discretization of stochastic partial differential equations. ANZIAM J., 45(E):C1-C15, (2003).

[22] A. J. Roberts, Wei Wang. Macroscopic reduction for stochastic reactiondiffusion equations. Preprint, 2008, arXiv:0812.1837v1 [math-ph]

[23] M. Schmuck, M. Pradas M, S. Kalliadasis, G. A. Pavliotis, New Stochastic Mode Reduction Strategy for Dissipative Systems, Physical Review Letters, 110:244101, (2013). 